\title{
Robust Photometric Stereo in a Scattering Medium via Low-Rank Matrix Completion and Recovery
}

\author{
Hao Fan*, Yisong Luo*, Lin Qi*, Nan Wang*, Junyu Dong*, Hui $\mathrm{Yu}^{\dagger}$ \\ * Ocean University of China,Qingdao, China \\ ${ }^{\dagger}$ University of Portsmouth, Portsmouth, UK \\ dongjunyu@ouc.edu.cn \\ hui.yu@port.ac.uk
}

\begin{abstract}
Photometric stereo is a popular method for its better detail recovery. However, when it is used in a scattering medium such as lake and ocean, the recovery result will be impacted negatively by the absorption, scattering and the impurities in water. In this paper, we present a new method to solve the problem of better $3 D$ reconstruction via LowRank Matrix Completion and Recovery . First, we use the dark points like shadows and darkness in water to fit the scattering effect distribution and then remove the scattering from the image. Next, we use robust principal component analysis method (RPCA) to recover the image by removing the sparse noise including shadows, impurities and some corrupted points caused by backscatter compensation. Finally, we combine the RPCA results and least-squares(LS) results to get the normal and accomplish the 3D reconstruction. Extension experimental results demonstrate that our method achieves accurate estimates of surface normal and 3D reconstruction than previous techniques.
\end{abstract}

\section{Introduction}

Remote Operated Vehicle(ROV) is the commonest technique to explore underwater environment, and providing better human system interaction between operators and ROV is significant to breakthrough. As a important part of ROV, imaging system makes it easier for operators to intuitively control this complex robotic system [1]. Further, the most important mission of imaging system is to transform the 2D images taken by submarine into 3D scene, i.e. 3D reconstruction.

There are many optical techniques for 3D reconstruction [2], such as photometric stereo, structure light, binocular stereo vision, and structure from motion. Photometric stereo recovers 3D shape from different viewpoints with more than three different artificial illuminations [3], [4], [5]. Structure light [6] based on the triangulation principle is mostly used in active techniques in underwater systems, which scans the surfaces with a spot or a stripe of light and is effective in distant and close range acquisitions. Binocular stereo vision [7] and structure from motion [8] are mostly used for passive techniques which are typically used in close and medium range acquisition.

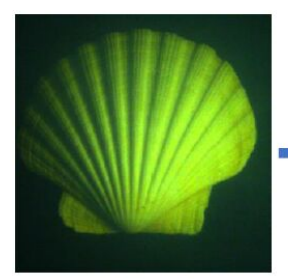

(a) Original

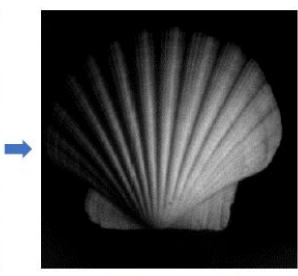

(b) De-scattered

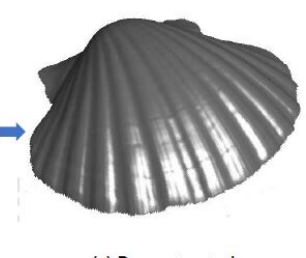

(c) Reconstructed
Figure 1. The procedure of our reconstructing method. Original image is transform into de-scattered image, and then is reconstructed to 3D depth map by photometric stereo via RPCA.

In this paper, we focus on photometric stereo, which can produce higher-quality geometry than others. However, when applying photometric stereo in scattering medium, the measured light carries information not only about the scene orientation but also the medium itself [9]. In underwater environment, lights get attenuated and scattered by the particles of the medium. So, the light which has entered the camera may or may not have been reflection by the object, i.e. the direct or scatter component. The scatter component is the most unwanted signal which adds a strong negative influence to $3 \mathrm{D}$ reconstruction. In other words, the formulation of photometric stereo is supposed to be linear, however, affected by backscatter which attenuates underwater light exponentially with distance, which makes the traditional solving method fail [10].

However, this paper present a new method to deal with the influence of backscatter in scattering medium. concretely, we first compensate backscatter, which is similar to the method presented in [11]. And after the measured backscatter is subtracted from the input image, the formulation of photometric stereo in scattering medium becomes linear so that we can estimate depth, surface normal and albedo as original photometric stereo does. However, some noise still exist due to the errors caused by removing backscatter, shadows and impurities in medium etc., which makes it not as significant as normal. So we use the RPCA method [12] to recover the low-rank property of image matrix so that we can improve the accuracy of surface normal and albedo [13]. Further, we can get higher-quality geometry. Fig.1 shows the procedure of our method. 
We demonstrate our results through extensive experiments in a water tank with a special device. We evaluate the performance of our method in different backscatter levels. It can be seen from the results that the recovered shapes are similar to those in clear water even at high levels of water turbidity.

\section{Related Work}

In this section, we briefly review some related works for photometric stereo used in scattering environment. When photometric stereo method is used in underwater environment, the light propagation model is changed, because of the light attenuation and scattering by the medium.

A large amount of work [9], [14] has focused on modelling image formation with diffuse illumination in underwater environment or sub-sea. The light which has entered the camera may or may not have been reflection by the object. There are three components in the light path as shown in Fig.2: a) The light which enters camera without reflection from the object is called backscatter; b) Light which reflected by the object and has not been scattered in the scattering medium, called the direct component; c) Light which reflected by the object but has been small angle scattered, called the forward-scattered component.

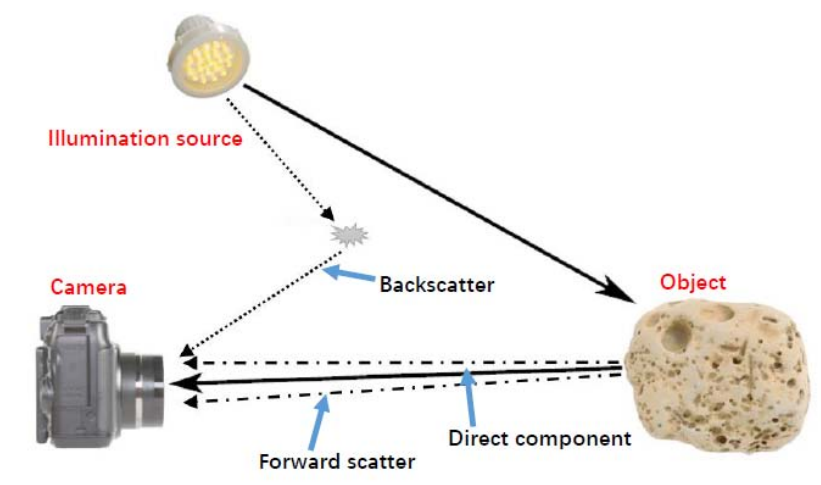

Figure 2. Light Propagation Model. The model combines three components: the direct component; the forward scatter component; and the backscatter component.

Photometric stereo with backscatter: Narasimhan et al. [15] formulated photometric stereo model which deals with backscatter and attenuation, with the supposition of distant light sources and an orthographic camera. The method relies on solving a system of non-linear equations, however, the optimization does not always converge and final solution is highly dependent on the initial approximation [10]. Murez, Treibitz et al. [16] considered both backscatter and forward scatter, and formulates a considerable complex model which cannot be solved directly but approximatively.

Instead of compensating for the backscatter effect, some previous systems use hardware setup to minimize the effect of backscatter. In [17], the authors used special polarizing hardware to recover the image. These methods in [17], [18] can reduce the effect of backscatter, but cannot completely remove it. Besides, the designed hardwares of these methods, were mostly more complex and expensive.

Tsiotsios et al. [11] put forward a new method to compensate effectively for the backscatter with three point light sources, in which way it becomes a linear formulation of photometric stereo as in the air. In this work, the authors used the dark points to fit the backscatter distribution, which is proved useful to remove the effect of backscatter component.

Photometric stereo with RPCA: Wu et al. [13] presented a new approach to robustly solve the photometric stereo problem. They considered photometric stereo as a one of recovering a low-rank matrix from missing and corrupted observations such as shadows and specularities. Unlike previous approaches that used least-squares techniques, they used an advanced convex optimization technique [12], [19] that is guaranteed to find the correct low-rank matrix by simultaneously fixing its missing and erroneous entries.

\section{Light Propagation Model}

In this section, we introduce the light propagation model in the scattering medium. When light propagates in the scattering medium, it is attenuated exponentially with distance. And when the light comes from an artificial source, its intensity $I_{0}$ is inversely proportional to the travelled distance $d$ due to Inverse Square Law (ISL) defined as:

$$
I=\frac{I_{0}}{d^{2}} e^{-c d},
$$

where $I$ is the received intensity, and $c$ is the total extinction coefficient of the medium which describes the light loss per unit distance. The total extinction coefficient includes the mediums absorption $a$ and scattering coefficient $b$ as $c=a+b$ [14], where $b$ reflects the superposition of all scattering incidents around a unit volume particle and can be calculated by integrating the angular scattering function of the medium $\beta(\theta)$ over all the directions around the particle: $b=2 \pi \int_{0}^{\pi} \beta(\theta) \sin \theta \mathrm{d} \theta$, where $\theta$ is the angle between the original ray and scattered ray. We employ the low-order representation of [15] defining $\beta(\theta)$ as $\beta(\theta)=b / 4 \pi(1+g \cos \theta)$, where $g \in(-1,1)$.

Considering the artificial point light and camera are both put in a dark environment without other light sources, all illumination $L_{0}$ which camera receives comes from the artificial point light contains 3 components: 1) the direct component; 2) the backscatter component; and 3) the forwardscattered component. The three components represent an image:

$$
L_{0}=L_{d}+L_{b}+L_{f},
$$

where $L_{d}$ is the direct component, $L_{b}$ is the backscatter component, and $L_{f}$ is the forward scattering component. In our experiment, the effect of the forward scattering component is negligible, because the distance between the imaged object and camera is small enough $(0.6-1 \mathrm{~m})$. 


\subsection{Direct Component}

The direct component is the light direct reflect from the object. We assume that the surface is Lambertian with a spatially varying albedo $\rho$, and the size of the imaged object is small enough compared to the object distance from the camera, such that we can use orthographic projection approximations [11]. Then, we define the direct component as [3]:

$$
L_{d}=I(d) \frac{\rho}{\pi} \boldsymbol{l}_{\boldsymbol{k}} \boldsymbol{n}
$$

where $I(x)$ is the light comes from a pint source, and $\boldsymbol{n}$ is the unit normal vector of the imaged object. The light direction is denoted by unit vector $\boldsymbol{l}_{\boldsymbol{k}}$. Considering the attenuation described Eq.(1), the incident component in the underwater environment defined as $I(d)=\frac{I_{k}}{d_{1}^{2}} e^{-c d_{1}} e^{-c d_{2}}$, where $d_{1}$ is the distance from light to the imaged object, and $d_{2}$ is the distance between the imaged object and the camera.

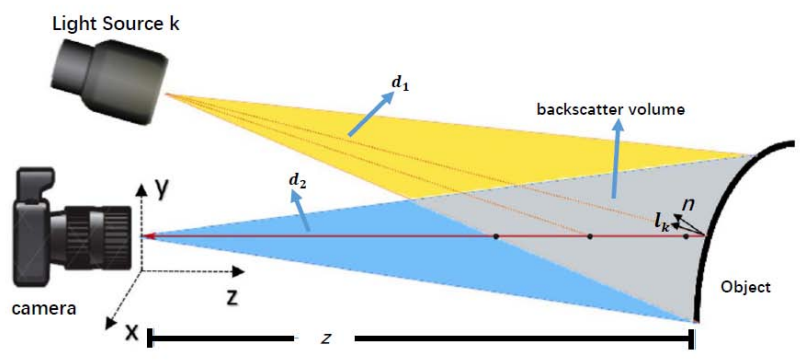

Figure 3. Geometry of light propagation. In our experiments, the measured brightness is the sum of the direct component and the scatter components from a point source.

Hence, the total direct component is:

$$
L_{d}=\frac{I_{k}}{d_{1}^{2}} e^{-c\left(d_{1}+d_{2}\right)} \frac{\rho}{\pi} \boldsymbol{l}_{\boldsymbol{k}} \boldsymbol{n} .
$$

\subsection{Backscatter Component}

Consider a differential scattering volume at distance $z$ across the Line-of-Sight (LOS) of the sensor pixel. As above, the light volume is defined as

$$
I(z)=\frac{I_{k}}{d_{1}(z)^{2}} e^{-c d_{1}(z)} e^{-c d_{2}(z)}
$$

According to the phase function $\beta(\theta)$, the light is scattered into each direction. Then the differential backscatter component that reaches the sensor pixel is $\mathrm{d} L_{b}=\frac{b I_{k}}{4}(1+$ $g \cos \theta) \frac{e^{-c d_{1}(z)}}{d_{1}(z)^{2}} e^{-c d_{2}(z)}$. The total backscattered light can be integrated over all depth distance $z$ along the pixels LOS. Since we use point light source, the integrate path is different for each pixel. For one pixel, we consider the path from $z_{k}$ to $z_{o}$, where $z_{k}$ is the start point and $z_{o}$ is the point on the object [11]. The total backscatter component in one pixel is given by

$$
L_{b}=\int_{z=z_{k}}^{z=z_{o}} \frac{b I_{k}}{4 \pi}(1+g \cos \theta) \frac{e^{-c d_{1}(z)}}{d_{1}(z)^{2}} e^{-c d_{2}(z)} \mathrm{d} z .
$$

This is a non-linear function, and there is no closed-form solution for the integral. The method in [15] tried to solve the non-linear equations, but the work assumed the light is not a real point source, and the optimization algorithm did not always converge. In this paper, we assume the imaged objects have the small variation surface, so that the backscatter does not depend on the unknown height of the objects and is a constant smooth distribution [17].

\section{Backscatter Decomposition}

\subsection{Backscatter Removal for PS}

The light propagation model in the scattering medium is defined as: $L_{0}=L_{d}+L_{b}$. As we all know, the extra ambiguity is mostly caused by the backscatter term. If we estimate the backscatter component correctly and remove it from the measured brightness, we can easily estimate the normal from the direct component:

$$
L_{0}-L_{b}=\frac{I_{k}}{d_{1}^{2}} e^{-c\left(d_{1}+d_{2}\right)} \frac{\rho}{\pi} \boldsymbol{l}_{\boldsymbol{k}} \boldsymbol{n} .
$$

In this paper, we assume the scale of object is much smaller than that of the distance between object and camera, and assume the point sources surround the camera at the same height. Because $d_{1}$ and $d_{2}$ is approximately equal for each pixel from different light sources, $\delta(x)=$ $\frac{1}{d_{1}^{2}} e^{-c\left(d_{1}+d_{2}\right)} \frac{\rho}{\pi}$ can be a constant value for each imaged object point. As a result,

$$
L_{d}=I_{k} \delta(x) \boldsymbol{l}_{\boldsymbol{k}} \boldsymbol{n} .
$$

The direct illumination appears linear, so the surface normal and various albedos can be estimated.

\subsection{Backscatter Estimation}

As we said before, the backscatter is a non-linear function, so it is hard to solve directly. Tsiotsios et al. [11] proposed a simple method for backscatter removal with fitting the backscatter as a binary quadratic function which can be fitted with six input points. They showed the energy of backscatter is not always positive correlative with the distance, which will saturate with a certain length. The respective direct component in dark points such as shadows and remote points will be 0 in which case $L_{0}=L_{b}$. By searching the local dark point in image, the backscatter can be estimated. Fig.4 shows the quadratic function fitted by our method and the orginal and de-scattered image of a shell.

In our method, we divide the image into numerous patches, and search all dark points in each patch which is smaller than a threshold value. Then, we randomly choose one dark point from each patch and use a binary quadratic 


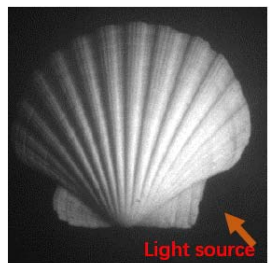

(a)

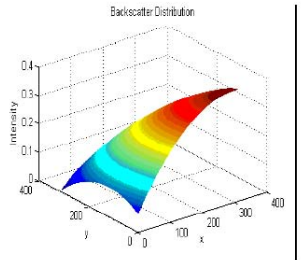

(b)

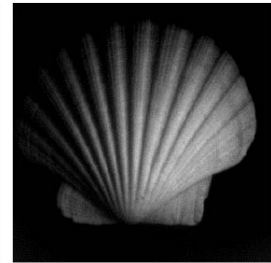

(c)
Figure 4. (a) The original image taken in the scattering medium $(150 \mathrm{ml}$ milk). (b) The scatter component fitted by dark points. (c) The de-scattered image.

function to estimate the distribution, which is different from [11] which only chose six darkest points. Since different solutions can be obtained with various selections of the local dark points above, we use a Random Sample Consensus (RANSAC) scheme to find the solution with minimum error, and make sure all the values of dark points are larger than the fitted value. This method is proved to be effective for estimating the distribution of backscatter, without any prior information.

\section{RPCA based Photometric Stereo}

When using a binary quadratic function to estimate the backscatter, it may bring some errors in pictures. Besides, shadows and impurities may also cause negative effects on the normal estimation and $3 \mathrm{D}$ reconstruction. We use an advanced convex optimization technique [12] to solve the problem, which is guaranteed to find the correct low-rank matrix by simultaneously fixing its missing and erroneous entries.

\subsection{Low-rank Property of Image Matrix}

In section 3, we mention to estimate the distribution of scattering component, and remove it from the original brightness. Then the left component is direct part $L_{d}$. Consider the matrix $\boldsymbol{O} \in \Re^{m \times n}$ constructed by stacking all the vectorized images, denoted by $\operatorname{vec}\left(L_{d k}\right)$, as

$$
\boldsymbol{O}=\left[\operatorname{vec}\left(L_{d 1}\right)\left|\operatorname{vec}\left(L_{d 2}\right)\right| \cdots \mid \operatorname{vec}\left(L_{d} n\right)\right]
$$

where $\operatorname{vec}\left(L_{d k}\right)=\left(L_{d k}(1), \cdots, L_{d k}(n)\right)^{T}$ for $k=$ $1, \cdots, n(n \geq 3)$. It follows Eq.(7) that $\boldsymbol{O}$ can be factorized as follows:

$$
\boldsymbol{O}=\boldsymbol{N L}
$$

where $(N)=\left[I_{1} \delta_{1}(x) \boldsymbol{n}_{\mathbf{1}}\left|I_{2} \delta_{2}(x) \boldsymbol{n}_{\mathbf{2}}\right| \cdots \mid I_{m} \delta_{m}(x) \boldsymbol{n}_{\boldsymbol{m}}\right]^{T}$, and $L=\left[\boldsymbol{l}_{1}\left|\boldsymbol{l}_{2}\right| \cdots \mid \boldsymbol{l}_{n}\right]$.Clearly the rank of the matrix $\boldsymbol{O}$ is at most 3, irrespective of the number of pixels $m$ and the number of images $n$.

\subsection{Efficient Solution based Low-Rank Matrix Completion}

Due to the shadows, impurities and some corrupted caused by backscatter removing, the direct component is not the ideal low-rank structure like $\boldsymbol{O}$. Thus, instead of Eq.(9), the image measurements should be:

$$
\boldsymbol{O}=\boldsymbol{N} \boldsymbol{L}+\boldsymbol{E},
$$

where matrix $\boldsymbol{E}$ accounts for corruption by shadows and specularities. Suppose that after backscatter is removed, only a small fraction of the pixels in each image is corruption and the most pixels are illuminated by the light source. Then, most pixels in the input images obey the low-rank diffusive model, and most entries in the error matrix $\mathrm{E}$ will be zero. So we can decompose $\boldsymbol{O}$ as the sum of a low-rank matrix and a sparse error matrix [20]. Thus, we can solve the above problem by finding a sparse matrix $\boldsymbol{E}$ such that the matrix $\boldsymbol{F}=\boldsymbol{N} \boldsymbol{L}$ has the lowest possible rank.

Using a Lagrangian formulation, we rewrite the above problem as the following optimization problem:

$$
\min _{\boldsymbol{F}, \boldsymbol{E}} \operatorname{rank}(\boldsymbol{F})+\gamma\|\boldsymbol{E}\|_{0} \quad \text { s.t. } \quad \boldsymbol{O}=\boldsymbol{F}+\boldsymbol{E},
$$

where $\|\cdot\|_{0}$ denotes the $l_{0}$-norm (number of non-zero entries in the matrix), and $\gamma>0$ is a parameter that trades off the rank of the solution $\boldsymbol{F}$ versus the sparsity of the error $\boldsymbol{E}$. But it is not tractable since both rank and $l_{0}$-norm are non-convex and discontinuous functions. Then use convex relaxation to recover the low-rank matrix $\mathrm{F}$ from corrupted observations $\boldsymbol{O}$ :

$$
\min _{\boldsymbol{F}, \boldsymbol{E}}\|\boldsymbol{F}\|_{*}+\gamma\|\boldsymbol{E}\|_{1} \quad \text { s.t. } \quad \boldsymbol{O}=\boldsymbol{F}+\boldsymbol{E},
$$

Where $\|\cdot\|_{*}$ and $\|\cdot\|_{1}$ represent the nuclear norm and $l_{1}$ norm, and $\gamma>0$ is a regularization parameter.

Thenwe use the Augmented Lagrange Multiplier (ALM) method which is called Robust PCA to solve the problem. Let $(\hat{\boldsymbol{F}}, \hat{\boldsymbol{E}})$ be the optimal solution to Eq.(12). And with the given light direction $L$, we can easily recover the matrix $N$ of surface normal from $\hat{\boldsymbol{F}}$ as:

$$
\boldsymbol{N}=\hat{\boldsymbol{F}} \boldsymbol{L} \text {. }
$$

Actually, the most area of the object can accord with the assumption of low-rank matrix and the sparse error matrix. But some points with larger gradient may have more shadows in most images, and don't accord with the assumption. These points can hard correctly get the normal of imaged object. To solve the problem, we propose a method that we segment the image to two parts, that one is the points with less shadows using the robust RPCA method to solve, and the other with more shadows using the least-squares(LS) method. Then, we combine the two method to recover the normal and build the 3D reconstruction [21], [22]. Fig.5 shows the sparse noises and the normal map computed by RPCA.

\section{Experiments}

\subsection{Experimental Setup}

Our experimental setup is shown in Fig.6, which is put in front of a black water pool, with all the lights in the water. 


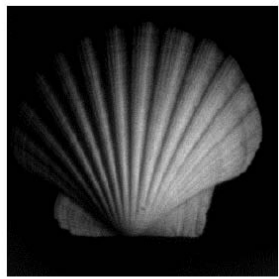

(a)

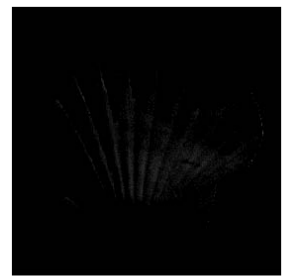

(b)

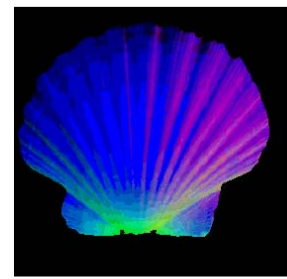

(c)
Figure 5. (a) De-scattered image. (b) Spare noises removed by RPCA. (c) Normal map computed by RPCA.

We use IDS UI-358xCP-C camera which placed $5 \mathrm{~cm}$ away from the water to take pictures, and use underwater LED lights with white illumination to light the imaged objects. All the lights are placed in a rotating circular orbit around the camera, with the same slant angle and different tilt angles. The objects were placed at an average distance of $60 \mathrm{~cm}$ from the camera. The size of imaged object is within $5-10 \mathrm{~cm}$, enforcing the orthographic assumption. We do the experiments in a black water pool in a dark room to make sure that all the lights the camera recieves are only from our light sources. We used tap water, and the turbidity was increased by adding milk (milk is nearly purely scattering).
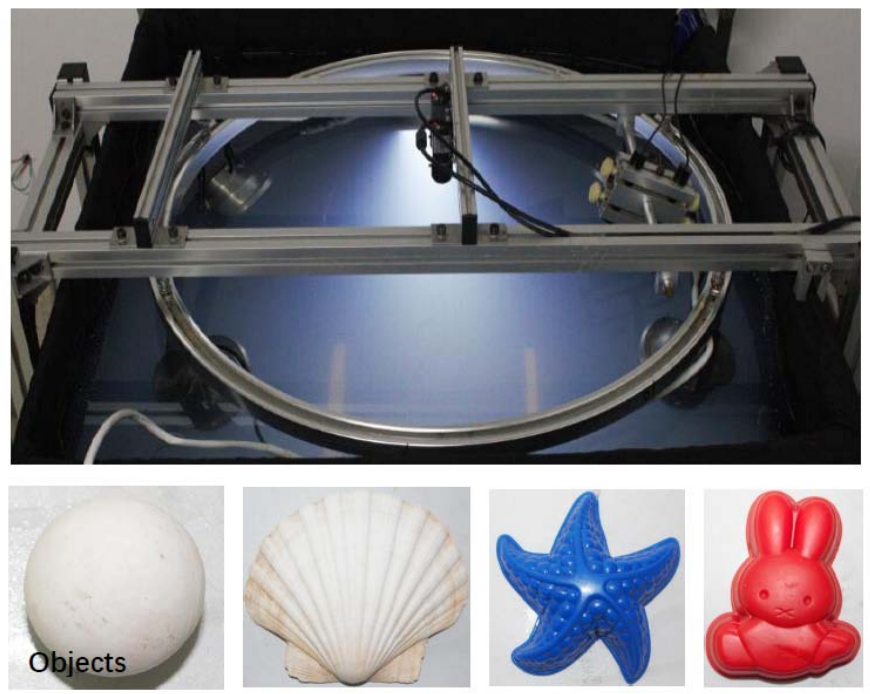

Figure 6. Experimental Device and Imaged Objects

We firstly evaluate our method with a standard sphere whose ground-truth normal maps are already known. We design several experiments to evaluate the correctness of our method by computing the angular errors between the estimated normal map and the ground-truth. We then evaluate our method in real objects such as shell and plastic toys to estimate the normal and reconstruct the $3 \mathrm{D}$ surfaces.
TABLE 1. MEAN ERROR (IN DEGREE)

\begin{tabular}{cccccc}
\hline turbidity $\left(\mathrm{ml} / \mathrm{m}^{3}\right)$ & 0 & 50 & 100 & 150 & 200 \\
\hline LS & 2.9902 & 2.6052 & 2.9363 & 3.3685 & 3.0463 \\
RPCA [13] & 3.0492 & 2.4616 & 3.3801 & 3.9015 & 4.5624 \\
Our method & $\mathbf{2 . 7 8 8 3}$ & $\mathbf{2 . 4 0 0 0}$ & $\mathbf{2 . 9 0 1 8}$ & $\mathbf{3 . 3 2 7 3}$ & $\mathbf{2 . 9 7 5 0}$ \\
\hline
\end{tabular}

TABLE 2. MEAN ERROR WITH DIFFRENT NUM. OF IMAGE IN 50 AND 100 TURBIDITY

\begin{tabular}{cccccc}
\hline $\begin{array}{c}\text { turbidity } \\
\left(\mathrm{ml} / \mathrm{m}^{3}\right)\end{array}$ & num. of images & 8 & 16 & 24 & 48 \\
\hline \multirow{2}{*}{50} & LS & $\mathbf{2 . 8 3 8 3}$ & $\mathbf{2 . 8 4 9 3}$ & 2.8939 & 2.9010 \\
& Our method & 31.4637 & 12.2012 & $\mathbf{2 . 6 3 2 0}$ & $\mathbf{2 . 5 8 6 3}$ \\
\hline \multirow{2}{*}{200} & LS & $\mathbf{4 . 2 7 0 3}$ & $\mathbf{3 . 7 0 6 6}$ & 3.3242 & 3.0463 \\
& Our method & 31.4145 & 11.8901 & $\mathbf{3 . 3 0 6 7}$ & $\mathbf{2 . 9 7 5 0}$ \\
\hline
\end{tabular}

\subsection{Quantitative Evaluation}

In this section, we use a sphere under different turbidity water to evaluate the performance of our method. The diameter of imaged Lambertian sphere is $3 \mathrm{~cm}$, which is small enough compared to the distance from the camera. It can be considered as orthographic projection. We employ the mean angular errors to evaluate the quality of our reconstruction. The mean angular error is defined as: $E=\operatorname{mean}\left(\arccos \left(N \cdot N_{g}\right)\right)$, where $N$ is the estimate normal, and $N_{g}$ is ground-truth. We consider three influence factors-turbidity, the number of the input images, and source light refraction.

6.2.1. The influence of turbidity. In this experiment, a sphere is placed at the center of tank, and we image the sphere under 48 lighting conditionswhere the slant angle of light directions is $45^{\circ}$. We increase the turbidity of water by adding $50 \mathrm{ml}$ milk each time into a 1 cubic meter tank. We employ three methods to estimate the normal and the Comparison results is shown in Table.1.

It can be seen that these three methods can all estimate the normal in an acceptable range after backscatter removal. When the turbidity increases, the values of the RPCA method become better than LS, but our method is always the best. It proves that our method can efficiently recover the surface normal in the turbidity water.

6.2.2. Effect of the number of the input images. In the above experiments, we have already used images of the object under 48 different illuminations. In this experiment, we want to find out the minimum number of images that our method can be better than LS method. The mean angle error is used as the measure of the estimate. We take experiments in two turbidity conditions with $50 \mathrm{ml}$ and $200 \mathrm{ml} \mathrm{milk}$, and the result is shown in Table.2. and Fig.7 more intuitively.

It is observed that our method is better than LS when the number of images is more than 24 whereas [13] requires at least 40 images. So the more images we input, the better result our method can get.

6.2.3. Effect of source light refraction. Since our method is proposed to used in real underwater envioronment, the 
TABLE 3. MEAN ERROR IN MILD TURBIDITY

\begin{tabular}{ccccccc}
\hline turbidity $\left(\mathrm{ml} / \mathrm{m}^{3}\right)$ & deviation & -2 & -1 & 0 & +1 & +2 \\
\hline \multirow{2}{*}{50} & LS & $\mathbf{2 . 5 2 7 8}$ & 2.5516 & 2.9010 & 3.3532 & 3.9387 \\
& Our method & 2.8769 & $\mathbf{2 . 4 8 1 0}$ & $\mathbf{2 . 5 8 6 3}$ & $\mathbf{2 . 6 5 7 1}$ & $\mathbf{3 . 1 1 2 3}$ \\
\hline \multirow{2}{*}{200} & LS & $\mathbf{3 . 7 0 6 4}$ & 3.9140 & 3.7471 & 4.0236 & 4.6897 \\
& Our method & 3.9204 & $\mathbf{3 . 8 9 5 5}$ & $\mathbf{3 . 4 3 4 7}$ & $\mathbf{3 . 6 6 3 5}$ & $\mathbf{4 . 3 3 4 2}$ \\
\hline
\end{tabular}

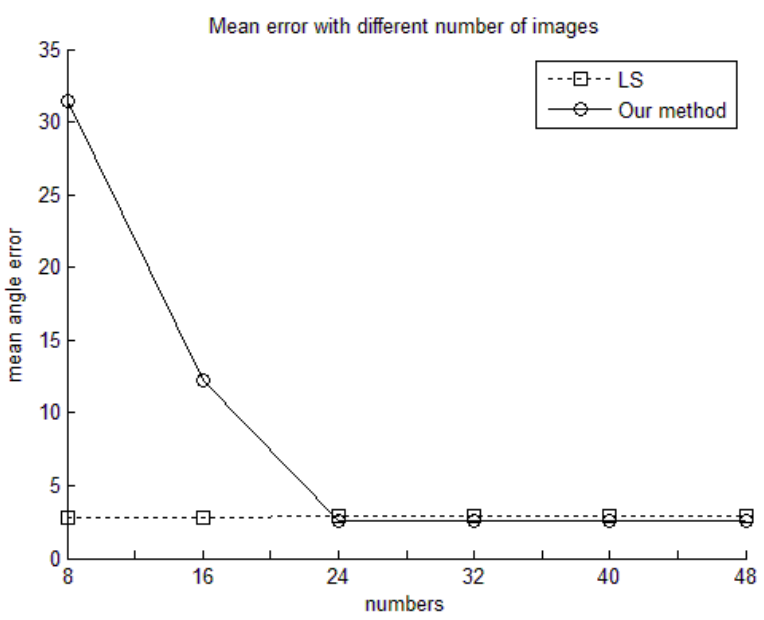

Figure 7. Mean error with different number of images. We compare our method with LS in scattering medium whose tubidity is $50 \mathrm{ml} / \mathrm{m}^{3}$.

effect of refraction cannot be ignored besides the scatter and absorb in water. We still use the mean angle errors to estimate the effect under 48 different light conditions. We take experiments in two turbidity conditions with $50 \mathrm{ml}$ and $150 \mathrm{ml}$ milk .

The result is shown in Table.3. We can observe that the mean angle error becomes bigger, if the light direction is changed. In an acceptable range, our method can get better result than $\mathrm{LS}$.

\subsection{The result of real objects}

We test our method in some real objects, including a plastic rabbit, a plastic starfish, and a real shell. We put the object at the center of the tank, and adjust the slant light direction into $45^{\circ}$. Then, we add $50 \mathrm{ml}$ milk each time into the tank, which makes the clear water turbid enough. Since the actual groundtruth normal and depth maps are not available for these object, we consider the results in clear water as groundtruth. We compare our results about varing levels of turbid water with groundtruth and the results computed by direct PS, as shown in Fig. 8 .

It is observed that our results in turbid water are similar to groundtruth. Compared to direct PS method, the geometry and the normal map we produced are more accurate than direct PS. Further more, our method provides more details. Taking the rabbit as the example, our method can reconstruct the area of eyes and mouth better where the depth is concave-convex. Obviously, our method can remove backscatter, deal with the errors such as shadows, impurities in water, and corrupted points caused by backscatter compensation, and successfully recover the 3D shape with more details.

\section{Conclusion}

In this paper, we presents a new computational method for robust photometric stereo in a scattering medium via Low-Rank Matrix completion and recovery. We use backscatter fitting method to remove the backscatter component, and then formulate photometric stereo problem as a rank minimization problem that can be solved efficiently by convex optimization. The biggest advantage of the proposed method is its ability to handle shadows, impurities, errors from backscatter estimation, and other kinds of largemagnitude, non-Gaussian errors in scattering water.

For these, our method can provide better 3D information in ocean exploration than previous works. But in our experiment, it needs more lights. In the future work, we focus on how to keep the robust results when reduce the number of light sources. Such effects can help to let the method be better used in a ROV or AUV for marine survey technology.

\section{Acknowledgments}

This work was supported by International Science\&Technology Cooperation Program of China(ISTCP) (No.2014DFA10410).

\section{References}

[1] S. Andrew, R. Fredrik, and C. Ryan, "An interactive interface for multi-pilot rov intervention," The Ocean's 16 Marine Technology Society(MTS), 2016.

[2] B. Mukundappa, N. S. Kumar, and R. P. Kumar, "Literature survey on building 3-d models of underwater objects," International Journal of Computer Science and Network Security (IJCSNS), vol. 12, no. 2, p. 89, 2012.

[3] R. J. Woodham, "Photometric method for determining surface orientation from multiple images," Optical engineering, vol. 19, no. 1, pp. 191 139-191 139, 1980.

[4] G. McGunnigle and J. Dong, "Augmenting photometric stereo with coaxial illumination," Computer Vision, IET, vol. 5, no. 1, pp. 33-49, 2011.

[5] J. Dong, G. McGunnigle, L. Su, Y. Fang, and Y. Wang, "Improving photometric stereo with laser sectioning," in Computer Vision Workshops (ICCV Workshops), 2009 IEEE 12th International Conference on. IEEE, 2009, pp. 1748-1754.

[6] C. Roman, G. Inglis, and J. Rutter, "Application of structured light imaging for high resolution mapping of underwater archaeological sites," in OCEANS 2010 IEEE-Sydney. IEEE, 2010, pp. 1-9. 


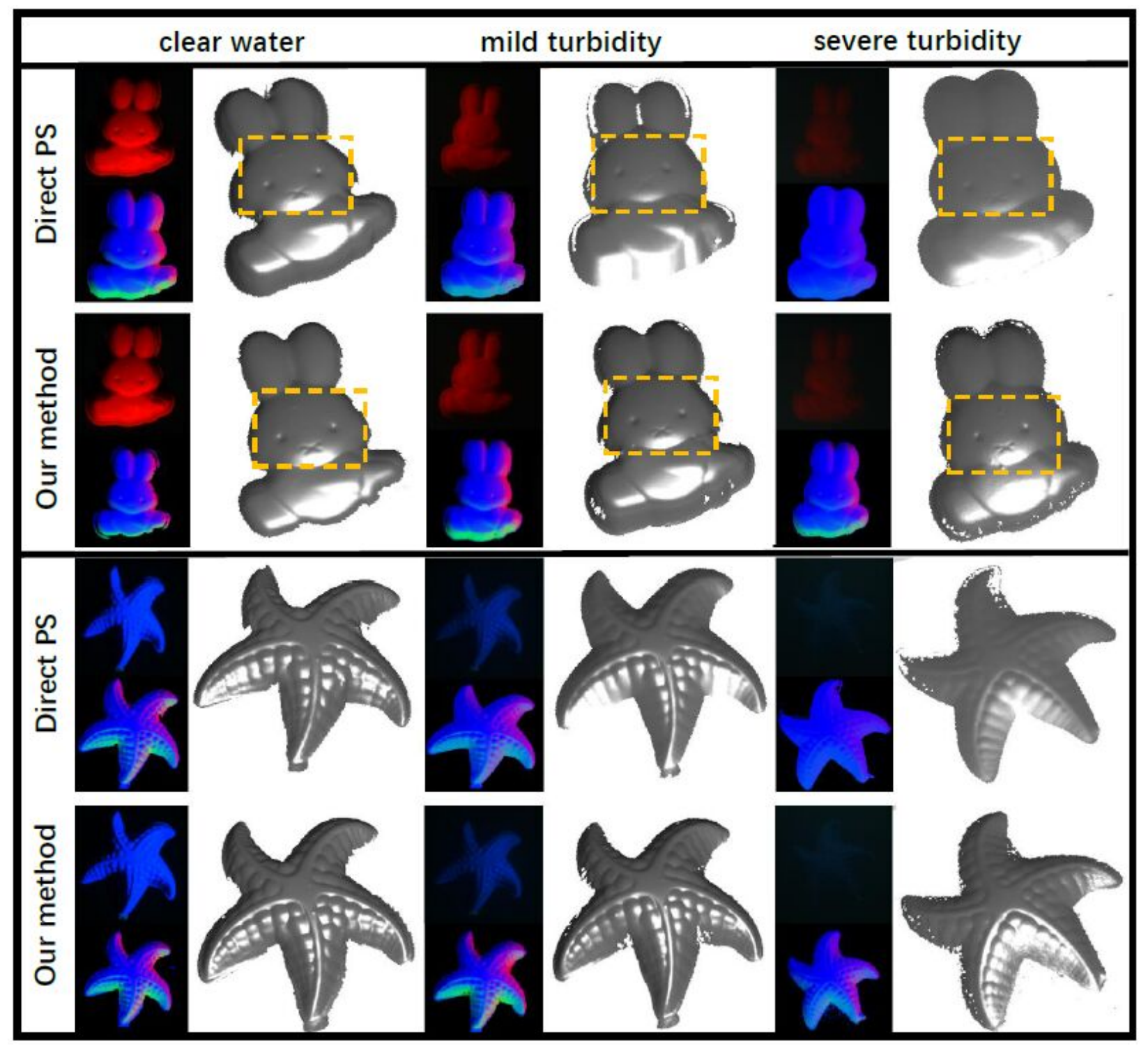

Figure 8. Shape reconstruction results in different scattering medium. We reconstruct a plastic rabbit and starfish with different tuibidity, and compare the results of our mothod with direct PS. Our method can provide more details and are more similar to the real objects in all tuibid water conditions.

[7] F. Bruno, G. Bianco, M. Muzzupappa, S. Barone, and A. Razionale, "Experimentation of structured light and stereo vision for underwater $3 \mathrm{~d}$ reconstruction," ISPRS Journal of Photogrammetry and Remote Sensing, vol. 66, no. 4, pp. 508-518, 2011.

[8] A. Sedlazeck, K. Köser, and R. Koch, "3d reconstruction based on underwater video from rov kiel 6000 considering underwater imaging conditions," in OCEANS 2009-EUROPE. IEEE, 2009, pp. 1-10.

[9] J. S. Jaffe, "Underwater optical imaging: the past, the present, and the prospects," Oceanic Engineering, IEEE Journal of, vol. 40, no. 3, pp. 683-700, 2015.

[10] M. E. Angelopoulou and M. Petrou, "Evaluating the effect of diffuse light on photometric stereo reconstruction," Machine vision and applications, vol. 25, no. 1, pp. 199-210, 2014.

[11] C. Tsiotsios, M. Angelopoulou, T.-K. Kim, and A. Davison, "Backscatter compensated photometric stereo with 3 sources," in Proceedings of the IEEE Conference on Computer Vision and Pattern Recognition, 2014, pp. 2251-2258.
[12] Z. Lin, M. Chen, and Y. Ma, "The augmented lagrange multiplier method for exact recovery of corrupted low-rank matrices," arXiv preprint arXiv:1009.5055, 2010.

[13] L. Wu et al., "Convex optimization based low-rank matrix completion and recovery for photometric stereo and factor classification," 2011.

[14] J. S. Jaffe, "Computer modeling and the design of optimal underwater imaging systems," Oceanic Engineering, IEEE Journal of, vol. 15, no. 2, pp. 101-111, 1990.

[15] S. G. Narasimhan, S. K. Nayar, B. Sun, and S. J. Koppal, "Structured light in scattering media," in Computer Vision, 2005. ICCV 2005. Tenth IEEE International Conference on, vol. 1. IEEE, 2005, pp. $420-427$.

[16] Z. Murez, T. Treibitz, R. Ramamoorthi, and D. Kriegman, "Photometric stereo in a scattering medium," in Proceedings of the IEEE International Conference on Computer Vision, 2015, pp. 3415-3423. 
[17] T. Treibitz and Y. Y. Schechner, "Active polarization descattering," Pattern Analysis and Machine Intelligence, IEEE Transactions on, vol. 31, no. 3, pp. 385-399, 2009.

[18] Y. Y. Schechner and N. Karpel, "Recovery of underwater visibility and structure by polarization analysis," Oceanic Engineering, IEEE Journal of, vol. 30, no. 3, pp. 570-587, 2005.

[19] E. J. Candès, X. Li, Y. Ma, and J. Wright, "Robust principal component analysis?" Journal of the ACM (JACM), vol. 58, no. 3, p. 11, 2011.

[20] L. Wu, A. Ganesh, B. Shi, Y. Matsushita, Y. Wang, and Y. Ma, "Robust photometric stereo via low-rank matrix completion and recovery," in Computer Vision-ACCV 2010. Springer, 2010, pp. 703-717.

[21] B. K. Horn, "Height and gradient from shading," International journal of computer vision, vol. 5, no. 1, pp. 37-75, 1990.

[22] A. Agrawal, R. Raskar, and R. Chellappa, "What is the range of surface reconstructions from a gradient field?" in Computer VisionECCV 2006. Springer, 2006, pp. 578-591. 